\title{
URGENCIAS GLOBALES, NECESIDADES LOCALES PARA INTERNACIONALIZAR LA UNIVERSIDAD PÚBLICA ARGENTINA. VACANCIAS EN MONITOREO Y MEDICIÓN DEL IMPACTO TERRITORIAL
}

\author{
Olga M. Saavedra* \\ Universidad Nacional de Río de Cuarto, Argentina \\ osaavedr@unr.edu.ar
}

Recibido: 31/07/2019 Aceptado: 4/11/2019

\section{Resumen}

El presente trabajo analiza críticamente la tensión existente entre la política pública argentina entre 2003 y 2015 en materia de internacionalización de la educación superior frente a las tendencias globales impuestas, y el aprovechamiento que las universidades públicas hacen en uso de su autonomía. En este marco se propone un indicador de internacionalización en función de la necesidad doméstica de fortalecer el sistema universitario nacional y se analiza en estos términos el impacto de los Programas Fortalecimiento de Redes Interuniversitarias y Misiones Universitarias al Exterior.

Los resultados de este artículo, se enmarcan en un trabajo de investigación mayor realizado para la obtención del doctorado de la autora, por lo que es coherente con el mensaje de la tesis: "la relación entre desarrollo territorial, universidad e internacionalización de la educación superior por su complejidad, no es lineal, ni establece un orden taxonómico entre sus términos. Tampoco es una sola, pues depende desde dónde se piensa en términos de espacio, territorio e historias; se reflexiona desde una perspectiva situada, asumiendo la posibilidad política de intervención, cambio y superación; de emancipación de aquella situación original en desventaja".

Palabras clave: Política pública argentina - Internacionalización de la educación superior Programa Redes Interuniversitarias - Programa Misiones Universitarias al Exterior.

\begin{abstract}
This paper critically analyses the tension between Argentine public policy between 2003 and 2015 regarding the internationalization of higher education against the imposed global trends, and the use that public universities make in using their autonomy. In this framework, this article proposes an indicator based on the domestic needs to strengthen the Argentinean university system and the impact of two emblematic programs of internationalization: Interuniversity's Networks and University's Missions Abroad.

The results of this article are framed in a major research work carried out to obtain the author's doctorate, so it is consistent with her thesis message: "the relationship between territorial development, university and internationalization of higher education is very complex, is not linear, nor does it establish a taxonomic order between its terms. It is not unique, because it depends on where you think in terms of space, territory and stories. Assuming a situated perspective, this relationship preserves the political possibility of emancipation of that disadvantageous situation at origin".
\end{abstract}

Keywords: Argentinean public policy - Internationalization of higher education - Program of Interuniversity's Networks - Program of University's Missions Abroad.

*Doctora en Desarrollo Territorial obtenido en la Universidad Nacional de Río Cuarto. Licenciada en Ciencia Política; Licenciada en Relaciones Internacionales, ambos grados académicos obtenidos en la Universidad Nacional de Rosario. 


\section{Contextos, temas y debates}

El tema 'internacionalización de la educación superior' (IlciónES) en principio, no tiene signo por delante. Requiere ser comprendido como un dato que se instala a partir de que las fronteras se vuelven más permeables y el concepto de distancia se relativiza como consecuencia en gran parte, de la tecnología que acerca el espacio físico inmaterializándolo en un espacio virtual. Antes de la revolución tecnológica las fronteras eran jurisdiccionales, el límite de lo nacional salvaguardaba el pensamiento nacional. La apertura al mundo que posibilitó el desarrollo de los dispositivos supuestamente para una más rápida y óptima comunicación, dejó al descubierto otros tipos de fronteras. Los territorios comprendidos casi exclusivamente desde la percepción geográfica empezaron a concebirse además en términos funcionales. La contigüidad física dejó de ser la única manera de generación de continuum.

En materia de educación las lógicas, las razones y los sentidos locales entraron en disputa con intereses globales y se empezó a poner en duda su propia naturaleza enraizada en Argentina desde el siglo XX en su concepción de derecho garantizado por el estado. A partir de la Reforma de 1918 las universidades se concibieron a sí mismas y alimentaron una cosmovisión desde su propia subjetividad: se veía y se admiraba al mundo desde esa ventana y había control de lo que se permitía entrar desde ese exterior. El exterior saltó la vieja y segura ventana no solo sin que el interior consiga impedirlo, sino que muchas veces retribuyó esa apertura con ventajas, beneficios y oportunidades atendibles. Por eso es que la globalización en materia de educación no tiene signo, ni es posible simplificar sus efectos generados sobre el sector, apenas en positivos y negativos. La política pública es la que cuenta para ello dotando a esa internacionalización de un rumbo y una dimensión valorativa para sus agentes.

El siglo XXI visibiliza a la educación como otro ámbito globalizado, y el desarrollo de políticas públicas que estimulan la proyección de la universidad en el mundo es reciente en Argentina. Es más bien un proceso, un camino a transitar en el que el colectivo universitario gestionado por el estado no puede pensarse ni expresarse unívocamente, no puede ser entendido ni mucho menos asumido como conjunto de personas que comparten características y se proponen objetivos comunes. La universidad es un territorio singular en sí misma, y si bien aquí no profundizamos en esta definición, aunque se hable en singular, la realidad universitaria se manifiesta contundentemente plural. Este proceso de relacionamiento institucional con pares del exterior, supone una gimnasia que -en principio- parece resultar más extraña a las universidades públicas que a las privadas pero que, en términos de relaciones académicas personales extendidas a los equipos de trabajo, constituye una práctica usual.

La internacionalización atraviesa todos los espacios de administración y gestión universitaria por lo que es necesario aplicar una lectura multidisciplinar y un ejercicio de doble enfoque cognitivo: uno macro-global y otro micro-institucional. La política pública nacional actuará como interfase entre ambos ajustes, por el territorialismo (Manzanal, 2008) o potestad y control del estado nacional sobre las Universidades Nacionales (UUNN), y la tensión que surge entre la capacidad de incidencia del estado nacional a través de sus políticas públicas y la autonomía universitaria. Ello motiva a que se sostenga que las universidades públicas son actores de cooperación académica descentralizada y no actores subnacionales de cooperación internacional.

La experiencia ganada en esta temática permite identificar las siguientes, como regularidades empíricas aplicadas a las UUNN en Argentina:

- Las acciones internacionales han sido promovidas por los individuos y equipos de investigación, antes que por el estado y las instituciones (aun cuando la formalización de la relación requirió siempre del aval institucional, tal lo que ocurre con los Convenios)

- Se observa mayor agilidad reactiva que capacidad proactiva. La oferta y la demanda externas condicionan pudiendo incluso desvirtuar la gestión en la producción del conocimiento y la definición de las agendas de investigación significativas para el propio territorio.

- Se constata mayor tendencia en concretar con pares institucionales extranjeros iniciativas de cooperación académica interuniversitaria que con pares argentinos.

- El insuficiente financiamiento desdibuja la intención de lo efectivamente viable.

Estas hipótesis particulares estarían contribuyendo a una hipótesis más general, más abarcadora y con fuerte responsabilidad en el devenir de la temática: la internacionalización de la universidad argentina ha sido objeto de inéditos esfuerzos y consecuentes avances de la política pública nacional entre 2003 y 2015 pero no logró consolidarse como política de estado.

¿Cómo hubiera sido posible que tales políticas de gobierno fragüen en "política sustentable de estado"? Puede haber más de una respuesta a esa pregunta. En principio, se puede decir que exigiría otro esfuerzo de reflexión pues abre una línea de trabajo a futuro, en gran parte dependiente de la reunión de estudios de caso. Pero también se puede responder -como sostiene el posicionamiento de este trabajo- que la transición entre una política de gobierno a una política de estado señala una tensión permanente que puede ser resuelta más rápidamente a nivel de los agentes, antes que esperarlo de la estructura.

Aun cuando la política pública nacional entre 2003 y 2015 ha estimulado los procesos de internacionalización de las universidades argentinas, no ha sido muy cuidadosa en explorar el impacto generado en los territorios nacionales o bien, los resultados no han sido difundidos quedando en todo caso, en órbitas tecnocráticas antes que políticas. A partir de 2016, lo registrado en la web de la SPU ha borrado todo vestigio de la memoria institucional. 
Tampoco se ha dado a conocer públicamente el presupuesto destinado a los programas de IlciónES debiéndose reconstruir a partir de los presupuestos destinados para el fortalecimiento de la Educación Superior o Programa 26.

\section{Redes para internacionalizar las IES argentinas. Programas emblemáticos}

La red es una dinámica de trabajo capaz de generar ese continuum funcional a objetivos o desafíos compartidos. El mecanismo privilegiado por la política de promoción de la universidad argentina (PPUA) en el exterior fue el de red. Las redes interuniversitarias fueron concebidas como alianzas estratégicas y estimuladas a expresarse bajo el formato de consorcios universitarios.

Las redes han sido una herramienta fundamental para construir vinculación directa y acercamiento entre profesionales dedicados a las funciones distintivas de una universidad: docencia, investigación y extensión. Se trata de la construcción de lazos que puedan trascender la esfera personal y convertirse en vínculos institucionales entre cátedras, carreras y facultades (...) Alentar estos procesos implica trabajar sobre la oferta académica, la calidad del servicio, las regulaciones sobre los intercambios (de personas, servicios, conocimientos e información), la imagen de la universidad en el exterior y las barreras culturales e idiomáticas, entre otras cuestiones de similar relevancia (SPU, mini-sitio web: 2006).

Si hay una convicción que atraviesa este capítulo es que Redes y Misiones -modo coloquial para referirse a los Programas Redes Interuniversitarias y Misiones Universitarias al Exterior implementadas por la política educativa nacional a partir de 2006- instalaron la necesidad de definir una política internacional propia de las instituciones de educación superior (IES) en las cúpulas de gestión y eso fue leído por las comunidades académicas como una oportunidad de movilizarse primero para luego, aspirar a objetivos de creciente envergadura.

Las Redes Interuniversitarias se pensaron para vincular dos o más universidades argentinas con pares del exterior. Las Misiones Universitarias al Extranjero en cambio, originalmente se pensaron para promover y aprender usos y prácticas en materia de gestión y administración académica internacional. Por ello, sus destinatarios principales eran decisores, que podían acelerar cambios en ese sentido: funcionarios rectorales, de las unidades académicas, directores de centros e institutos de investigación, sellos editoriales universitarios, entre otros. La Misión servía para el reconocimiento del territorio del socio potencial y concluía por lo general con la intención de la firma de un Convenio que se concretaba luego que se cumplían las formalidades legales al regreso de esa misión. En materia de gestión, se enfatizó en la necesidad de la adopción del crédito como unidad de medida para estudiantes, a los efectos de facilitar el reconocimiento de tramos cursados fuera de las universidades locales a quienes les resulta sumamente dificultoso lograr su reconocimiento de acuerdo a los estándares vigentes en el país. Por el contrario, los estudiantes extranjeros con una simple constancia -incluso emitida por el docente o supervisor académico- logran la homologación de los estudios realizados en Argentina, en su país de origen. Otro tópico explorado fue el de titulaciones conjuntas de posgrado.

Tanto Redes como Misiones compartieron dos rasgos: la condición de asociativismo y la de cofinanciamiento. El asociativismo era una condición válida para la consolidación del conjunto en un verdadero sistema universitario. El co-financiamiento refiere a que el estado nacional financiaba la mayor parte de la ejecución de los proyectos, pero las instituciones participantes debían contribuir con un porcentaje del monto solicitado que, si bien era mínimo, resultaba útil a la hora de analizarlo como estrategia de apropiación colectiva y requisito de transferencia al medio universitario y, en la medida de lo posible, al medio local.

Ambos programas, fueron sumamente utilizados por las universidades argentinas -públicas y privadas): del 2006 al 2015 hubo 1522 proyectos beneficiados -según los registros de las Resoluciones correspondientes a los resultados de cada Convocatoria. No podemos dar la cifra de total de proyectos postulados porque no ha sido información en principio disponible en los sitios oficiales, tampoco hay evidencia de que haya sido información relevada por las instancias de gobierno. ¿Cómo impactó este aprovechamiento de la política pública en el subsistema de universidades públicas? Para dar respuesta al interrogante surgió la necesidad de elaborar un indicador de rendimiento.

Desde el recupero de la democracia en Argentina, las Universidades también recuperaron el autogobierno. Tal recupero se inició bajo la analogía de la disputa político-partidaria que se daba en el terreno nacional. Este rasgo de algún modo, condicionó la diversidad y renovación en las gestiones institucionales por razones ideológicas y también de disponibilidad presupuestaria. Este contexto, estimuló que el destaque fuera puesto en algunas IES y no en otras. Ahora bien, si se tiene en cuenta que entre 2003 y 2015 se crearon 17 nuevas universidades de gestión estatal ${ }^{2}$ es válido preguntarse ¿'las nuevas' pudieron aprovechar este dispositivo de proyección internacional que ofreció la política pública?

\footnotetext{
${ }^{1}$ Ellas son las UU.NN. de Río Negro, Del Chaco Austral, Arturo Jauretche, en 2007; Avellaneda, Moreno, José C. Paz, Villa Mercedes, Del Oeste, en 2009; T. del Fuego, Antártida e Islas del Atlántico Sur, en 2010; de las Artes, Defensa Nacional, Hurlingham, Comechingones, Rafaela, en 2014; y Alto Uruguay, San Antonio de Areco y Scalabrini Ortiz, en 2015. En 2015 eran 55 IESP.
} 
¿Fueron integradas al resto? Pueden sumarse a estas dos inquietudes muchas otras, pero la gran pregunta de fondo tiene que ver con el monitoreo y la evaluación de la política de internacionalización implementada en Argentina entre 2003 y 2015. Quienes adquirimos experiencia en la gestión técnica de algunas instituciones intervinientes en estos programas y, además, estudiamos, analizamos e investigamos el tema, sabemos de la relevancia del monitoreo y la evaluación de las medidas y de las políticas. Como se sabe además del riesgo de una inserción dependiente al mundo global (Kreimer, 2006:205), es imprescindible establecer una evaluación en función de alguna variable de desarrollo doméstico o nacional. De este propósito, surge el IIDRD o Índice Internacional de Desarrollo Relacional Doméstico que se propuso mostrar cuán beneficiosos fueron Redes y Misiones, para el fortalecimiento de la trama de relaciones del sistema universitario nacional.

\section{La construcción de un indicador de internacionalización, en función de una necesidad nacional. EI IIDRD}

En la disputa que existe entre mandatos globales y necesidades locales, es posible tomar posición desde la conciencia de ser productores periféricos de conocimiento -que no es lo mismo que conocimiento periférico- respecto de los que marcan tendencia en el mundo, sin desistir de eventuales oportunidades ofrecidas por la cooperación internacional. La respuesta que intentamos fue la construcción de un indicador de internacionalización en función de una variable/necesidad nacional: el fortalecimiento del sistema universitario de gestión pública.

Para construir este índice, se analizaron los 1522 consorcios ganadores de universidades argentinas en los programas Redes y Misiones a lo largo de nueve convocatorias del Programa Redes, y siete convocatorias del Programa Misiones, en un periodo de nueve años. Lamentablemente, las Resoluciones de la SPU que fueron el medio de verificación y objeto sistematizado, adolecieron de múltiples vicios de confección y carencia de datos. No dejaron constancia de los socios extranjeros de las universidades e instituciones argentinas participantes por lo que hay aspectos de esta evaluación que han quedado vacantes. A menos que la denominación del proyecto contuviera el nombre de la institución extranjera, no hubo modo de considerar esta información. En tal sentido, fueron los proyectos del Programa Misiones al Exterior los que mayormente contenían referencias al destino de la respectiva misión en la denominación de los proyectos, lo que resultó de utilidad para analizar tendencias en términos de integración del Sector Educativo del Mercosur (SEM), para mencionar otro aspecto del estudio, pero que no será analizado en este trabajo.

La autonomía institucional es el límite de la política pública. El estado no puede obligar a que las universidades tomen un rumbo. Al respecto, un ex Ministro de Educación manifestó respecto del énfasis puesto por la política pública de aquel momento en el Programa ARFITEC (Argentina Francia, Formación de Ingenieros y Tecnología). "La política del estado nacional, en ese momento, priorizó la formación de ingenieros y destinó el financiamiento necesario en cooperación con el gobierno francés, para fortalecer una profesión sensible para el desarrollo del país. Fue un recurso puesto a disposición de las universidades argentinos, las que podían aceptarlo como no. Era su prerrogativa por activación de la autonomía universitaria. La prerrogativa del estado nacional era el diseño de una política pública que estimulaba esa área del conocimiento más que otras. (Entrevista a Daniel Filmus, CABA, 02-05-2016)

Este tipo de dispositivos concuerdan con la posición asumida en este estudio: por un lado, la indelegable responsabilidad que le cabe al estado nacional en la orientación de sus políticas de internacionalización del conocimiento y de las IES según propósitos de desarrollo nacional. Por otro lado, el énfasis puesto en este análisis en la dimensión de la agencia, y su capacidad transformadora, antes que esperar que el cambio solo fluya en sentido vertical.

De la sistematización de la información disponible, se pudo obtener un mapeo con la cantidad de vínculos inter-universitarios dentro del sistema nacional originados por los proyectos, por un lado, y el registro de relaciones inter-universitarias dentro del país, por otro. Es válido aclarar que no son categorías asimilables. La cantidad de vínculos que cada proyecto generó se relaciona con la cantidad de instituciones contenidas en cada consorcio y por lo tanto, su impacto en el fortalecimiento de esta trama del territorio. Así, un consorcio de dos universidades argentinas A y B genera un vínculo entre dos actores similares pero que adquiere una significación que puede ser distinta para cada institución; la pertenencia de ambas a un mismo consorcio marca un contacto entre ellas. Los vínculos, en realidad son la información que nos permite inferir el fortalecimiento del sistema nacional en términos de mutuo conocimiento y cooperación doméstica coordinada, más allá de que pueda o no, sostenerse en el tiempo. El propósito del estudio fue formulado en positivo: a) verificar que el proceso de internacionalización de universidades públicas consolide el subsistema de UUNN, y b) determinar una medida de su impacto. Por tanto, se puso el acento en la faz arquitectónica constructiva de la política pública nacional. Esto significa que en el presente artículo se dejó de lado el análisis de la faz deliberativa, así como de su faz agonal.

Por otro lado, el IIDRD permitió mapear la incidencia en términos de integración que Redes y Misiones tuvieron en el sistema universitario nacional.

Para ejemplificar el conteo de vínculos se muestra a continuación cómo una red integrada por 11 universidades públicas argentinas -como la red ICAARG (Instituto de Ciencia Animal de Cuba + universidades bajo el PPUA de Argentina)- puede aportar en densificar la trama de relaciones entre IESU a nivel doméstico y, por lo tanto, consolidar la totalidad del sistema. 
En términos de fortalecimiento de la trama del sistema público universitario, un consorcio conformado por 11 IES argentinas lo fortaleció 55 veces por las 55 vinculaciones de doble flujo entre ellas. En términos de relaciones inter-pares: hay 11 IES integradas en el sistema de UUNN, con una trama de 55 vinculaciones recíprocas. Lo que evidencia este indicador es que cada vez que cada universidad integraba un consorcio o proyecto con idénticas o diferentes pares, contribuía por un lado al fortalecimiento del sistema como tal, pero por otro a la integración del colectivo en el mismo, sobre todo, si se participaba a las universidades más nóveles. El mapeo de estas vinculaciones, también muestran preferencias de relacionamiento entre determinadas universidades lo que densifica la trama del sistema en determinadas partes del mapa, y la debilita en otras. (Figura 2.)

Figura 1. Conteo de las vinculaciones según el IIDRD para una red de 11 instituciones

\begin{tabular}{|c|c|c|c|c|c|c|c|c|c|c|}
\hline Univ. 1 & & & & & & & & & & \\
\hline $\operatorname{con} 2$ & Univ. 2 & & & & & & & & & \\
\hline $\operatorname{con} 3$ & $\operatorname{con} 3$ & Univ. 3 & & & & & & & & \\
\hline $\operatorname{con} 4$ & $\operatorname{con} 4$ & $\operatorname{con} 4$ & Univ. 4 & & & & & & & \\
\hline $\operatorname{con} 5$ & $\operatorname{con} 5$ & $\operatorname{con} 5$ & $\operatorname{con} 5$ & Univ. 5 & & & & & & \\
\hline $\operatorname{con} 6$ & $\operatorname{con} 6$ & $\operatorname{con} 6$ & con6 & $\operatorname{con} 6$ & Univ. 6 & & & & & \\
\hline $\operatorname{con} 7$ & $\operatorname{con} 7$ & $\operatorname{con} 7$ & $\operatorname{con} 7$ & $\operatorname{con} 7$ & $\operatorname{con} 7$ & Univ. 7 & & & & \\
\hline $\operatorname{con} 8$ & $\operatorname{con} 8$ & $\operatorname{con} 8$ & $\operatorname{con} 8$ & $\operatorname{con} 8$ & $\operatorname{con} 8$ & $\operatorname{con} 8$ & Univ. 8 & & & \\
\hline $\operatorname{con} 9$ & $\operatorname{con} 9$ & con 9 & $\operatorname{con} 9$ & $\operatorname{con} 9$ & con 9 & con 9 & $\operatorname{con} 9$ & Univ. 9 & & \\
\hline $\operatorname{con} 10$ & $\operatorname{con} 10$ & $\operatorname{con} 10$ & $\operatorname{con} 10$ & con 10 & $\operatorname{con} 10$ & con 10 & $\operatorname{con} 10$ & con 10 & Univ.10 & \\
\hline con 11 & con 11 & con 11 & con 11 & $\operatorname{con} 11$ & con 11 & con 11 & con 11 & con 11 & con 11 & Univ.11 \\
\hline $10+\downarrow$ & $9+\downarrow$ & $8+\downarrow$ & $7+\downarrow$ & $6+\downarrow$ & $5+\downarrow$ & $4+\downarrow$ & $3+\downarrow$ & $2+\downarrow$ & $1=\nabla$ & 55 \\
\hline
\end{tabular}

Fuente: elaboración propia

De la aplicación del mecanismo recién descripto para cada uno de los 1522 proyectos o consorcios sistematizados y seleccionados durante el tiempo en que tanto Redes como Misiones estuvieron vigentes, surge el siguiente orden de contribución al fortalecimiento del sistema doméstico, propuesto como función dependiente de ambos dispositivos de internacionalización.

Tabla1. Cantidad de vinculaciones generadas por cada IES pública argentina según orden de contribución al fortalecimiento del sistema doméstico de ES argentino vía los programas de internacionalización Redes y Misiones 2006-2015

\begin{tabular}{|l|l|l|l|}
\hline \multicolumn{1}{|c|}{ IES } & $\begin{array}{c}\text { Cantidad de } \\
\text { Vinculaciones } \\
\text { en 9 ediciones } \\
\text { de REDES }\end{array}$ & \multicolumn{1}{c|}{ IES } & $\begin{array}{c}\text { Cantidad de } \\
\text { Vinculaciones } \\
\text { en 7 ediciones } \\
\text { de MISIONES }\end{array}$ \\
\hline U. Córdoba & 287 & U. Litoral & 132 \\
\hline U. La Plata & 261 & U. Córdoba & 126 \\
\hline U. de Rosario & 250 & UBA & 105 \\
\hline UBA & 217 & U. Quilmes & 103 \\
\hline U. Litoral & 202 & U. La Plata & 96 \\
\hline U. Río IV & 183 & U Río IV & 90 \\
\hline U. Nordeste & 154 & U. Rosario & 89 \\
\hline U. Gral. Sarmiento & 144 & U. Gral. Sarmiento & 85 \\
\hline U. Tucumán & 142 & U. San Luis & 84 \\
\hline U. Quilmes & 128 & U. San Martín & 82 \\
\hline U. Cuyo & 127 & U. Lanús & 70 \\
\hline
\end{tabular}




\begin{tabular}{|c|c|c|c|}
\hline UNICEN & 118 & U. Cuyo & 61 \\
\hline U. S. Luis & 116 & UNICEN & 57 \\
\hline U. S. Martín & 112 & U. La Pampa & 55 \\
\hline UTN & 111 & U. V. María & 53 \\
\hline U. La Pampa & 107 & U. del Sur & 50 \\
\hline U. Mar del Plata & 102 & U. Jujuy & 49 \\
\hline U. del Sur & 94 & U. Comahue & 46 \\
\hline U. Lanús & 93 & U. Tucumán & 46 \\
\hline U. Entre Ríos & 92 & UNNOBA & 41 \\
\hline U. Jauretche & 91 & U. Jauretche & 41 \\
\hline UNNOBA & 88 & U. Nordeste & 40 \\
\hline U. Luján & 81 & U. La Matanza & 39 \\
\hline U. Comahue & 79 & U. Sgo. del Estero & 39 \\
\hline U. S. Juan & 78 & U. Entre Ríos & 38 \\
\hline U. V. María & 75 & UTN & 37 \\
\hline U. Jujuy & 73 & U. Mar del Plata & 37 \\
\hline U. Sgo. del Estero & 69 & U. S. Juan & 36 \\
\hline U. Salta & 68 & U. Tres de Febrero & 35 \\
\hline U. Catamarca & 63 & U. Patagonia Austral & 34 \\
\hline U. Misiones & 55 & U. Luján & 32 \\
\hline U. Moreno & 55 & U. R. Negro & 28 \\
\hline U. Avellaneda & 51 & U. Avellaneda & 25 \\
\hline U. La Rioja & 51 & U. Salta & 23 \\
\hline U. Tres de Febrero & 50 & U. Lomas de Zamora & 22 \\
\hline U. Chilecito & 50 & U. Catamarca & 19 \\
\hline U. Formosa & 48 & U. Misiones & 18 \\
\hline U. San Juan Bosco & 40 & U. San Juan Bosco & 14 \\
\hline U. Patagonia Austral & 38 & U. Chilecito & 14 \\
\hline U. La Matanza & 33 & U. Moreno & 9 \\
\hline U. Nac. de las Artes & 25 & U. Formosa & 9 \\
\hline U. Chaco Austral & 23 & U. Nac. De las Artes & 6 \\
\hline U. R. Negro & 21 & U. La Rioja & 6 \\
\hline U. Lomas & 18 & U. Tierra del Fuego & 3 \\
\hline U. Autónoma de E. Ríos & 11 & U. José C. Paz & 3 \\
\hline U. Tierra del Fuego & 7 & U. Pedagógica & 2 \\
\hline U. José C. Paz & 6 & U. Autónoma de E. Ríos & 1 \\
\hline U. Pedagógica & 2 & U. Chaco Austral & 0 \\
\hline U. V. Mercedes & 1 & Inst. de Est. Sup. del Ejérc & 0 \\
\hline Inst. de Est. Sup. del Ejérc & 0 & U. V. Mercedes & 0 \\
\hline U. Defensa Nacional & 0 & U. Defensa Nacional & 0 \\
\hline U. Alto Uruguay & 0 & U. Alto Uruguay & 0 \\
\hline U. Hurlingham & 0 & U. Hurlingham & 0 \\
\hline U. Comechingones & 0 & U. Comechingones & 0 \\
\hline U. Rafaela & 0 & U. Rafaela & 0 \\
\hline U. S. Antonio de Areco & 0 & U. S. Antonio de Areco & 0 \\
\hline U. del Oeste & 0 & U del Oeste & 0 \\
\hline U. Scalabrini Ortiz & 0 & U. Scalabrini Ortiz & 0 \\
\hline
\end{tabular}

Fuente: elaboración propia

A continuación, se muestra la cantidad de universidades con las que cada IES mantuvo al menos un contacto, por causa de los Programas Redes y Misiones, independientemente de la cantidad de proyectos o consorcios en los que cada una participó. Entre paréntesis se consigna el año respectivo de creación.

Tabla 2. Índice de contribución de cada IES pública argentina a la integración del sistema doméstico vía los programas de internacionalización Redes y Misiones, entre 2006 y 2015

\begin{tabular}{|l|c|}
\hline \multicolumn{1}{|c|}{ IES Pública } & IDRD \\
\hline Universidad Nacional de Córdoba (1613) & 42 \\
\hline Universidad de Buenos Aires (1821) & 40 \\
\hline Universidad Nacional de Rosario (1968) & 40 \\
\hline
\end{tabular}


REVISTA DE LA ESCUELA DE CIENCIAS DE LA EDUCACIÓN, AÑO 16, NRO. 15, VOL. 2, JULIO A DICIEMRE DE 2020. PÁGINAS 68-80. ISSN 2362-3349 (EN LÍNEA). URGENCIAS GLOBALES, NECESIDADES LOCALES PARA INTERNACIONALIZAR LA UNIVERSIDAD PÚBLICA ARGENTINA. VACANCIAS EN MONITOREO Y MEDICIÓN DEL IMPACTO TERRITORIAL. OLGA M. SAAVEDRA

\begin{tabular}{|c|c|}
\hline Universidad Nacional de La Plata (1889) & 39 \\
\hline Universidad Nacional de Cuyo (1939) & 37 \\
\hline Universidad Nacional del Litoral (1919) & 36 \\
\hline Universidad Nacional Gral. Sarmiento (1993) & 35 \\
\hline Universidad Nacional de San Luis (1973) & 34 \\
\hline Universidad Nacional de Río Cuarto (1971) & 34 \\
\hline Universidad Nacional del Centro de la Pcia. de Buenos Aires (1974) & 34 \\
\hline Universidad Nacional del Nordeste (1956) & 34 \\
\hline Universidad Nacional de La Pampa (1973) & 33 \\
\hline Universidad Nacional de Lanús (1995) & 32 \\
\hline Universidad Tecnológica Nacional (en conjunto) (1959) & 31 \\
\hline Universidad Nacional del Comahue (1965) & 31 \\
\hline Universidad Nacional de Jujuy (1973) & 31 \\
\hline Universidad Nacional del Noroeste de la Pcia. de Buenos Aires (2002) & 30 \\
\hline Universidad Nacional de Entre Ríos (1973) & 30 \\
\hline Universidad Nacional de Tucumán (1914) & 30 \\
\hline Universidad Nacional de Quilmes (1989) & 29 \\
\hline Universidad Nacional de Salta (1972) & 29 \\
\hline Universidad Nacional Gral. San Martín (1992) & 28 \\
\hline Universidad Nacional de Luján (1973) & 28 \\
\hline Universidad Nacional de la Patagonia Austral (1995) & 28 \\
\hline Universidad Nacional de Mar del Plata (1962) & 28 \\
\hline Universidad Nacional de Santiago del Estero (1973) & 28 \\
\hline Universidad Nacional del Sur (1956) & 26 \\
\hline Universidad Nacional de Tres de Febrero (1995) & 26 \\
\hline Universidad Nacional de Río Negro (2007) & 25 \\
\hline Universidad Nacional de La Matanza (1989) & 25 \\
\hline Universidad Nacional Arturo Jauretche (2009) & 24 \\
\hline Universidad Nacional de la Patagonia San Juan Bosco (1980) & 24 \\
\hline Universidad Nacional de Villa María (1995) & 24 \\
\hline Universidad Nacional de Catamarca (1972) & 24 \\
\hline Universidad Nacional de San Juan (1973) & 23 \\
\hline Universidad Nacional de Formosa (1988) & 23 \\
\hline Universidad Nacional de Chilecito (2002) & 21 \\
\hline Universidad Nacional de Misiones (1973) & 21 \\
\hline Universidad Nacional de La Rioja (1993) & 20 \\
\hline Universidad Nacional de Avellaneda (2009) & 18 \\
\hline Universidad Nacional de Moreno (2009) & 17 \\
\hline Universidad Nacional de Lomas de Zamora (1972) & 16 \\
\hline Universidad Nacional de las Artes (2014) & 14 \\
\hline Universidad Nacional del Chaco Austral (2007) & 11 \\
\hline Universidad Autónoma de Entre Ríos (2012) & 8 \\
\hline Universidad Nacional de Tierra del Fuego, Antártida e Islas del Atlántico Sur (2010) & 8 \\
\hline Universidad Nacional de José Clemente Paz (2009) & 6 \\
\hline
\end{tabular}




\begin{tabular}{|l|c|}
\hline Universidad Nacional de Villa Mercedes (2009) & 1 \\
\hline Universidad Pedagógica Nacional (2013) & 1 \\
\hline Instituto de Enseñanza Superior del Ejército (1990) & 0 \\
\hline Universidad de la Defensa Nacional (2014) & 0 \\
\hline Universidad Nacional Alto Uruguay (2015) & 0 \\
\hline Universidad Nacional de Hurlingham (2014) & 0 \\
\hline Universidad Nacional de los Comechingones (2014) & 0 \\
\hline Universidad Nacional de Rafaela (2014) & 0 \\
\hline Universidad Nacional de San Antonio de Areco (2015) & 0 \\
\hline Universidad Nacional del Oeste (2009) & 0 \\
\hline Universidad Nacional Raúl Scalabrini Ortiz (2015) & 0 \\
\hline
\end{tabular}

Fuente: elaboración propia

Cada número de la columna que acompaña al listado de las universidades nacionales argentinas, representa la cantidad de instituciones nacionales con las cuales la universidad respectiva estableció vinculación en el marco de Redes y Misiones. Por ejemplo, en el caso de la Universidad Nacional de Rosario, UNR -institución de pertenencia de la autora del presente artículo- ésta generó vinculación con 40 IES de gestión pública argentina, sobre un universo de 58.

La finalidad de consignar el año de creación, es agregar el argumento probable de que de las nueve instituciones que quedan 'fuera' del sistema, en gran parte se deba a su condición de nóveles, pero también deben estar incidiendo otras causas por lo que se presentan casos como los de la Universidad Nacional de Oeste, así como del Instituto de Enseñanza Superior del Ejército, imponiéndose profundizar en las razones de esta situación. El año consignado para la UADER, así como para la Universidad Pedagógica y el Instituto Superior del Ejército son aquellos en los que eligió autoridades rectorales por primera vez por claustro de decanos y fueron reconocidos como instituciones superiores nacionales, respectivamente.

A continuación, se presenta la Figura 2. Mapa con el nivel de integración de la IES públicas argentinas por Redes y Misiones entre 2006 y 2015, de elaboración original para la Tesis Doctoral de la autora. En la última columna denominada "Otras" se consignan la cantidad de vinculaciones establecidas con universidades o instituciones de gestión privada y otros organismos del sistema público.

Para su mejor comprensión señalamos las siguientes referencias. El eje horizontal superior lista el nombre de las IES y el vertical su sigla en idéntico orden. Se tuvieron en cuenta todas las instituciones de educación superior universitarias o IESU públicas, existentes al año 2015. Las celdas vacías indican ausencia de relación entre las instituciones definidas por tales coordenadas. En las celdas que contienen 2 números, el primero refiere a la cantidad de proyectos de Redes compartidos y el segundo a Misiones. Las celdas que contienen un solo número se distinguen por el color; los verdes corresponden a Redes y los rojos a Misiones La frontera inferior del dibujo que se forma, se compone por una línea de celdas distinguidas en color amarillo. Los números contenidos en cada una de ellas representan el riesgo en generar el efecto contrario a la consolidación del sistema nacional universitario pues representa las veces que esa IES emprendió un proyecto sin la confluencia de un par argentino -ya sea de gestión pública o privada- y, lo grave consiste en que contó con la aprobación y financiamiento del estado nacional a través de la administración de la SPU. Finalmente, las zonas destacadas en gris constituyen la periferia del sistema pues son aquellas universidades e institutos que no tuvieron la posibilidad de participar de los programas analizados y, por ende, no usufructuaron estos dispositivos de internacionalización de la política pública nacional.

\section{Análisis}

Hay un $15,5 \%$ del total del sistema que permanece desintegrado en términos domésticos.

En las Bases de Redes, consta la siguiente definición: "Un proyecto de Red estará conformado por al menos tres (3) Instituciones Universitarias. Las Instituciones Universitarias extranjeras serán al menos un tercio (1/3) del total de las participantes" (Bases Redes VI). Requisito que no cambia hasta la última convocatoria analizada. Esta formulación dice mucho. Primero define tres como mínimo número requerido de instituciones para conformar una red; segundo expresamente define en un tercio, la participación de entidades extrajeras para conformar esa red. Sin embargo, la expresión al menos, abrió la posibilidad de ponderar el vínculo con instituciones extranjeras antes que con argentinas, no obstante esas mismas bases exigían que existieran convenios de cooperación académica con las universidades extranjeras asociadas. 


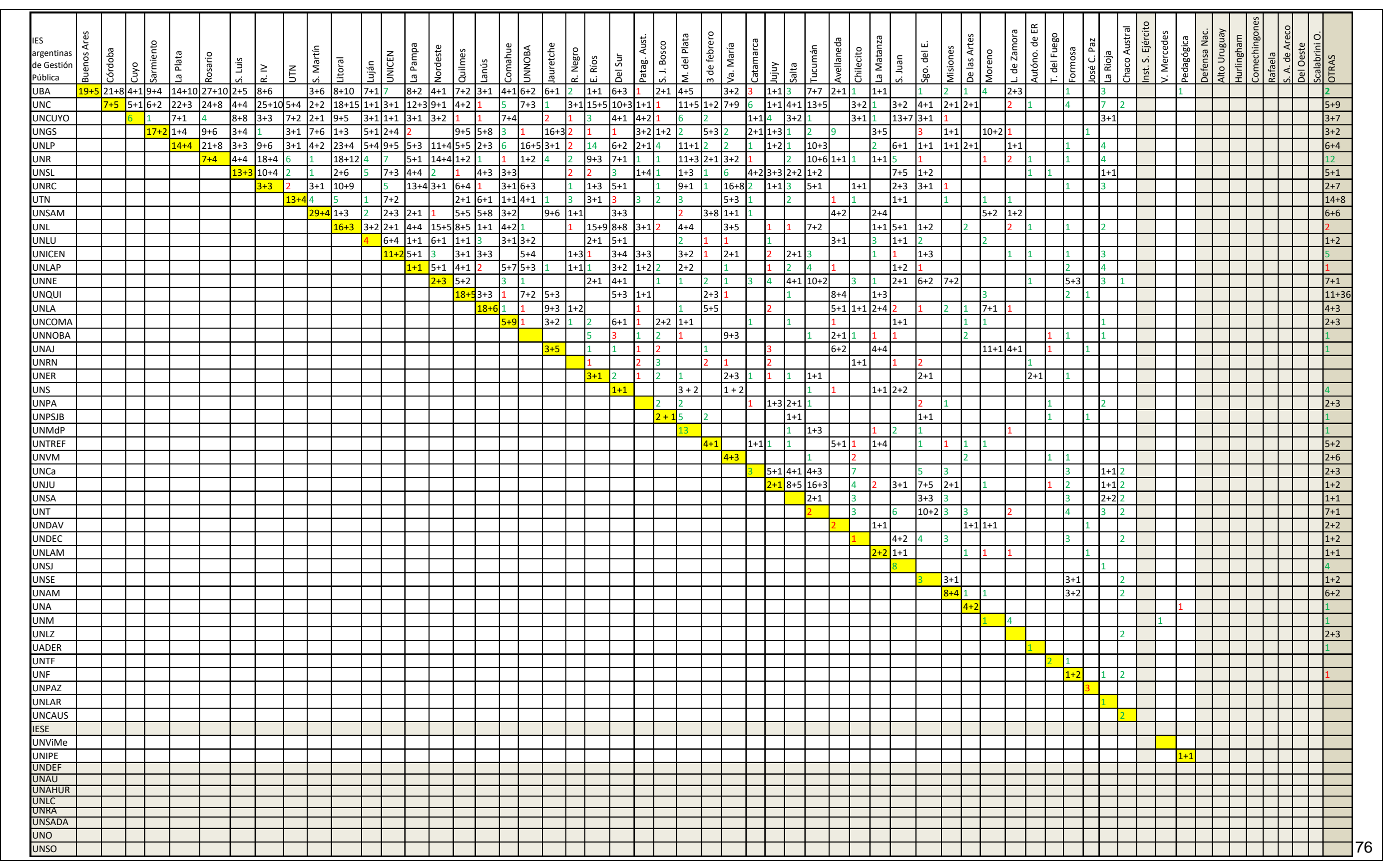


El caso de Misiones es sensiblemente distinto por lo que la incidencia de los números en la diagonal amarilla, claramente configura una desviación de los efectos procurados por el Programa.

Ello es así porque en las Bases de Misiones correspondientes a la convocatoria de 2008, constan exigencias categóricas como: "Cada proyecto deberá ser una presentación conjunta de al menos cuatro (4) instituciones universitarias argentinas $y / 0$ instituciones de reconocido prestigio científico, tecnológico o artístico, gobiernos y/o empresas. En ningún caso el número de instituciones universitarias argentinas podrá ser inferior a tres (3)". En las Bases de Misiones V, se baja la cifra de 4 a 3 instituciones argentinas; en Misiones VI se disminuye aún más y se exigen " $(\ldots)^{2}$ o más instituciones universitarias argentinas..." y en la última convocatoria considerada en este estudio, Misiones VII, desaparece: "Cada proyecto deberá ser una presentación de una o más instituciones universitarias argentinas. El proyecto será presentado al PPUA por una de las universidades argentinas asociadas, denominada al efecto "Universidad convocante".

En consecuencia, y atendiendo las bases establecidas, en todas las convocatorias de Redes y Misiones para promocionar las universidades argentinas, hubo instituciones autorreferenciadas, es decir, que presentaron proyectos unilaterales sin la confluencia de otra institución argentina, graficadas en la diagonal destacada en amarillo. Por lo tanto, la expresión universidad autorreferenciada alude a aquella IES que propuso un proyecto de Misión o Red al exterior sin generar previamente asociación con un par argentino.

La universidad más autorreferenciada en Redes fue UNSAM con 29 proyectos; y la más autorreferenciada en Misiones fue UNCOMA con 9 proyectos sin socia nacional.

En Misiones VII, se debilita la conducta asociativa. De 127 proyectos seleccionados, 84 son liderados por una sola universidad argentina, lo que significa una desviación del espíritu original del Programa equivalente al $64,5 \%$ de los proyectos evaluados y beneficiados por la política pública nacional. Tal lo ya explicado, en Misiones VII desaparece el requisito de asociativismo: una -o más instituciones universitarias- podían liderar un proyecto de Misiones al Extranjero. Esa es la explicación -al menos en parte- al 64, 5\% de desviación. La mayor densidad de la trama de relaciones tendidas entre instituciones argentinas en función de Redes y Misiones se encuentra concentrada en aproximadamente el $29 \%$ de todo el sistema de universidades públicas, sin que ninguna de ellas haya conectado con la totalidad de las 58 registradas al 2015 y en ninguna de las 16 convocatorias analizadas.

La Plata, Litoral, Rosario, Río Cuarto, Córdoba, UTN (sin diferenciar entre Facultades Regionales) y General Sarmiento fueron las beneficiarias de la mayor cantidad de proyectos seleccionados en distintas convocatorias de Redes.

Apenas el $10 \%$ de instituciones analizadas cumplieron con la condición de asociativismo con un par argentino.

En la próxima tabla mostramos dimensión del financiamiento para estos programas de internacionalización, es decir la incidencia de ambos Programas Redes y Misiones en el Programa 26, incluido en el Presupuesto de la Jurisdicción 70, correspondiente al Ministerio de Educación.

Tabla 3. Incidencia presupuestaria de los Programas Misiones y Redes, en el Programa para el Desarrollo de la Educación Superior (P26)

\begin{tabular}{|l|l|l|l|r|}
\hline Año & M/P.26 (en \%) & R/P.26 (en \%) & (M+R)/ P26 (en \%) & PROGRAMA 26 (en \$) \\
\hline 2007 & 0,024 & 0,036 & $\mathbf{0 , 0 6}$ & 4.440 .176 .713 \\
\hline 2008 & & 0,044 & $\mathbf{0 , 0 4 4}$ & 5.781 .624 .249 \\
\hline 2009 & 0,023 & 0,042 & $\mathbf{0 , 0 6 5}$ & $\mathbf{8 . 3 0 1 . 0 3 1 . 0 2 2}$ \\
\hline 2010 & & 0,028 & $\mathbf{0 , 0 2 8}$ & 10.774 .688 .145 \\
\hline 2011 & 0,015 & 0,035 & $\mathbf{0 , 0 5}$ & $\mathbf{1 3 . 8 0 9 . 2 5 4 . 5 9 2}$ \\
\hline 2012 & & 0,033 & $\mathbf{0 , 0 3 3}$ & $\mathbf{1 8 . 2 0 4 . 7 9 2 . 7 2 5}$ \\
\hline 2013 & ${ }^{*} 0,016$ & 0,026 & $\mathbf{0 , 0 4 2}$ & 23.019 .991 .238 \\
\hline 2014 & & 0,025 & $\mathbf{0 , 0 2 5}$ & $\mathbf{3 1 . 7 8 8 . 0 8 8 . 0 8 7}$ \\
\hline 2015 & ${ }^{*} 0,018$ & 0,031 & $\mathbf{0 , 0 4 9}$ & $\mathbf{4 2 . 1 0 7 . 4 5 4 . 6 3 4}$ \\
\hline
\end{tabular}

Fuente: Elaboración propia

Desde fines de 2006 y hasta 2015, la incidencia de los programas Redes y Misiones del PPUA en el presupuesto destinado al Programa 26 fue inferior al $0,1 \%$

\footnotetext{
${ }^{2}$ Ambos dispositivos se fundamentan en el PIESCI que se formuló en 2003, y fue el Programa de Internacionalización de la ES y Cooperación Internacional, plan maestro del proceso de internacionalización de las instituciones de educación superior argentinas que estuvo a cargo de la Secretaría de Políticas Universitarias.
} 


\section{Conclusiones y otras reflexiones}

La insuficiencia o ausencia de indicadores y evaluación oficiales aplicados al colectivo universitario en general y de gestión pública en particular, han estimulado la construcción individual e interpretación unilateral de indicadores de internacionalización por parte de las instituciones, sin análisis de impacto sobre sus respectivos territorios de influencia, neutralizando nuevas políticas o ajustes de las que se encuentran vigentes.

La vinculación universidad pública-desarrollo territorial ha sido una relación asimilada a alianzas estratégicas con actores del sector productivo tanto formal como no formal, pero recurrentemente disociada del proyecto-territorio (Madoery: 2018, 125) y de las políticas locales, nacionales y regionales en el marco de la tensión respecto de las fuerzas globales dominantes.

Se corrobora la hipótesis de que se prefiere generar vínculos con pares del exterior antes que con pares del propio sistema. Esto hubiera requerido de acciones compensatorias de la política pública nacional o institucional para que el 'desvío' o debilidad en los efectos asociativos de los Programas Redes y Misiones se revirtiera.

Finalmente, y con respecto al financiamiento de ambos programas, el dinero que un país destina para instalar y consolidar una política pública es indicador de la voluntad que le imprime a ese objetivo. Cuando se alude a la voluntad política, resulta muy difícil evaluarla según a qué se aplique. En este caso, todo lo que se hizo a partir del 2003 fue de gran aporte porque había muy poco o casi nada antes de estas medidas en materia de internacionalización de la educación superior, específicamente en términos de política pública de alcance federal. Sin embargo, también es verdad que las cifras expresadas en la Tabla 3. denotan escasa voluntad de sostener o consolidar el rumbo tomado, y esa debilidad se expresa en que posteriormente al 2016, ninguno de estos programas fue sostenido a pesar de la buena respuesta recibida.

En las condiciones del sistema de UUNN argentinas constatadas en 2015 y aun en las actuales, resulta pertinente aplicar la 'pedagogía de la pregunta'. Si se focaliza el $15 \%$ de instituciones desintegradas o no incluidas en los más emblemáticos dispositivos de internacionalización de la ES como fueron Redes y Misiones, implementados de una manera innegablemente novedosa entre 2006 y $2015^{(2)}$ ¿es estratégica la decisión de integrarse al mundo antes que consolidar el sistema nacional, o desatender el nivel de desintegración que reviste? Tal vez algunos respondan que sí, motivados en la urgencia del tema; tal vez otros respondan que no, argumentando necesidades y prioridades. Lo que se constata es la ausencia de debate a nivel nacional y, seguramente, también se trata de un tema poco debatido a nivel de las instituciones. Lo que se señala entonces, es un déficit de participación en las definiciones políticas. Este estado de la cuestión motiva una nueva pregunta ¿la participación, forma parte de una metodología estratégica? ¿Sería oportuno que la política pública nacional considere este dato a los efectos de evaluar y realizar ajustes de las bases de tales programas, antes que deshabilitarlos? ¿La autonomía de las universidades está operando como elemento eficaz a la hora de delinear una política institucionalinternacional? ¿Se atiende las dinámicas sistémicas que atraviesan el escenario nacional, además del regional y global a la hora de planificar? ¿Los objetivos de desarrollo y relaciones externas responden solo a la coyuntura o se planifica también para el mediano y largo plazo? ¿Es genuina la lucha de las universidades públicas argentinas, por la defensa de su accesibilidad, gratuidad y carácter democrático?, o con mayor precisión, ¿es genuino el acompañamiento que afirman sus cúpulas políticas en su discurso público, en la lucha por esta causa de sus docentes, investigadores, estudiantes y administrativos?

La tensión entre la formulación y consolidación de una política pública, por un lado, y la autonomía de las universidades por otro, de alguna manera representa la tensión entre las teorías macro y micro sociales. Mientras las primeras dan cuenta del contexto global, dominado por el predominio del capitalismo que muestra su cara neoliberal más extrema, las segundas dan cuenta del aquí y del ahora.

En el marco del presente análisis, la agencia o actor en el que se focaliza este estudio es la institución universitaria, específicamente la universidad pública argentina. El contexto que la contiene visibiliza con claridad los dos modelos en pugna que hoy se manifiestan con toda tirantez: la universidad como derecho social, humano, inclusivo garantizado por el estado versus la universidad empresa, que vende el conocimiento que administra y que produce como un bien más de consumo en el mercado. Si bien la universidad no es el único territorio que monopoliza la producción del conocimiento, el producto casi con exclusividad que se le reconoce a la universidad es el conocimiento. La distribución asimétrica de poder que en el momento actual caracteriza al sistema mundo-moderno (Wallerstein, 1998), hace que la dimensión educativa de la globalización caracterice a la sociedad como sociedad del conocimiento y que se le asigne a ese producto más que un valor, un precio y creciente rentabilidad. Esta discusión deviene en otra, más de raíz filosófica y epistemológica y de matriz global: por un lado, el capitalismo académico, que se exterioriza en transformaciones, usos y costumbres de instituciones académicas, actores académicos y vinculaciones generadas entre la academia y el medio no- académico. Por otro, el capitalismo cognitivo que se interioriza en las personas, y transforma al propio sujeto cognoscente y que decide.

La universidad como actor político está cambiando. La universidad pública en Argentina también, a pesar de eslóganes y discursos principistas y reformistas. La autonomía y autarquía que en Argentina tiene la universidad, deben interpretarse como medios para alcanzar fines-otros; son potencias para transformar y lograr objetivos. Esa capacidad transformadora que tiene la universidad pública está 
relacionada directamente con su potencia política y con su posibilidad de construir capacidades endógenas. La Declaración de la Conferencia Regional -CRES- de Cartagena de Indias en 2008, ha significado una enorme plataforma de acuerdos entre gobiernos de la región y, a su vez, resultó ratificada recientemente en la CRES 2018 de Córdoba en lo que a esta línea de investigación reafirma: la educación es un derecho social bajo responsabilidad y garantía del estado nacional.

Entonces, ¿para qué internacionalizar la universidad? Se pueden dar varias y diversas respuestas a esta pregunta. Este trabajo propone apenas una. El estado nacional es responsable por su rumbo, así como cada institución en ejercicio de su autonomía es responsable de sus propias definiciones y metodologías para concretarlas. La internacionalización de la educación superior es un campo de confluencia multidisciplinar en el que la ciencia política y las relaciones internacionales deben acordar un maridaje equilibrado, que conduzca a la economía y no a la inversa. Con idéntica responsabilidad, el análisis internacional con respecto a este tema, debe asumir un posicionamiento situado, anclado en la propia realidad, pero con una perspectiva sistémica y con abordaje territorial para lograr una visión geopolítica que sea estratégica en la producción y gestión del conocimiento. Y la estrategia no puede ignorar la participación de las comunidades con sus necesidades endógenas y expectativas postergadas. Las apenas acciones desarticuladas de 'relaciones internacionales de las universidades' deben potenciarse en verdaderas políticas internacionales institucionales que fortalezcan la totalidad del sistema nacional para poder fraguar en políticas públicas sostenibles en el tiempo. Esto exige con la misma intensidad, mecanismos y dispositivos de monitoreo y evaluación que garanticen los ajustes necesarios no solo a los acomodamientos sistémicos multinivel sino a los objetivos programáticos y visión en el largo plazo que cada institución haya definido de sí misma.

\section{Referencias Bibliográficas}

Kreimer, P. (2006). ¿Dependientes o integrados? La ciencia latinoamericana y la nueva división internacional del trabajo. En: Nómadas (Col), (24), pp. 199-212 Disponible en:<http://www.redalyc.org/articulo.oa?id=105116598017> ISSN 0121-7550

Madoery, O. (2018). Reflexiones en torno a la planificación del desarrollo territorial. En: A. Rofman y A. García (Comp.) Planificación, región y políticas. En búsqueda de nuevas ideas para un proyecto productivo.; editado por Gaspar Herrero. Ciudad Autónoma de Buenos Aires: Universidad de Buenos Aires. Facultad de Ciencias Económicas.

Manzanal, M. (2008). Territorio, poder e instituciones. Una perspectiva crítica sobre la producción del territorio. En: M. Manzanal; M. Arzeno y B. Nussbaumer (Comp.). Territorios en construcción. Actores, tramas y gobiernos: entre la cooperación y el conflicto. Buenos Aires: CICCUS, pp. 15-50.

Programa de Internacionalización de la educación superior y cooperación internacional -PIESCI (2003), en "Cooperación internacional" En:http://portales.educacion.gov.ar/spu/cooperacion-internacional/

Saavedra, O. (2018). Tesis Doctoral "Desarrollo territorial, internacionalización de la educación superior y universidad pública argentina: desafíos, oportunidades, riesgos y vacancias en los inicios del siglo XXI"- UNRC. Documento Sin publicar.

Sitio de la SPU http://portales.educacion.gov.ar/spu/sistema-universitario/listado-de-universidades-e-institutos/

Wallerstein, I. (1998). Impensar las ciencias sociales. Siglo XXI: México, pp. 3-26.

\section{Resoluciones Programa PPUA}

576 SPU- 2007 Resultados Convocatoria denominada "Misiones Universitarias al Extranjero I" y asignación de fondos para su ejecución.

269 SPU- 2009 Resultados Convocatoria denominada "Misiones Universitarias al Extranjero II" y asignación de fondos para su ejecución.

1600 SPU- 2011 Resultados Convocatoria denominada "Misiones Universitarias al Extranjero III" y asignación de fondos para su ejecución.

2912 SPU- 2013 Resultados Convocatoria denominada "Misiones Universitarias al Extranjero IV" y asignación de fondos para su ejecución.

4027 SPU- 2013 Resultados Convocatoria denominada "Misiones Universitarias al Extranjero V" y asignación de fondos para su ejecución.

5276 SPU- 2015 Resultados Convocatoria denominada "Misiones Universitarias al Extranjero Vl" y asignación de fondos para su ejecución.

5246 SPU- 2015 Resultados Convocatoria denominada "Misiones Universitarias al Extranjero VII" y asignación de fondos para su ejecución.

210 SPU-28/12/2006 Resultados Convocatoria de "Proyectos de Fortalecimiento de Redes Interinstitucionales de las Universidades Argentinas" y asignación de fondos para su ejecución.

280 SPU- 26/03/2008 Resultados Convocatoria denominada "Fortalecimiento de Redes Interuniversitarias II" y asignación de fondos para su ejecución.

249 SPU- 20/03/2009 Resultados Convocatoria denominada "Fortalecimiento de Redes Interuniversitarias III" y asignación de fondos para su ejecución.

1492 SPU- 08/09/2010 Resultados Convocatoria denominada "Fortalecimiento de Redes Interuniversitarias VI" y asignación de fondos para su ejecución.

288 SPU- 29/12/2011 Resultados Convocatoria denominada "Fortalecimiento de Redes Interuniversitarias V" y asignación de fondos para su ejecución. 
2340 SPU- 21/12/2012 Resultados Convocatoria denominada "Fortalecimiento de Redes Interuniversitarias VI" y asignación de fondos para su ejecución.

207 SPU- 27/12/2013 Resultados Convocatoria denominada "Fortalecimiento de Redes Interuniversitarias VII" y asignación de fondos para su ejecución.

4426 SPU- 04/12/2014 Resultados Convocatoria denominada "Fortalecimiento de Redes Interuniversitarias VIII" y asignación de fondos para su ejecución.

2016-1968-E-APN-SECPU\#ME 24/10/2016 Resultados Convocatoria denominada "Fortalecimiento de Redes Interuniversitarias IX" y asignación de fondos para su ejecución. 